\title{
An alternative view on entropic measures applied to the nervous
} system

\author{
Diego Mateos [1,2,3 and Jose Luis Perez Velazquez ${ }^{4, *}$ \\ ${ }^{1}$ El Consejo Nacional de Investigaciones Científicas y Técnicas. CONICET. Argentina \\ ${ }^{2}$ Facultad de Ciencia y Tecnología. Universidad Autónoma de Entre Ríos (UADER). Oro Verde, Entre Ríos, \\ Argentina. \\ ${ }^{3}$ Instituto de Matemática Aplicada del Litoral (IMAL-CONICET-UNL). Santa Fe, Argentina. \\ ${ }^{4}$ The Ronin Institute, Montclair, NJ, 07043 USA; \\ ${ }^{*}$ Corresponding author: Jose Luis Perez Velazquez, jose.velazquez@ronininstitute.org, jlpvjlpv@gmail.com.
}

\begin{abstract}
In the same manner that there are several statements of the second law of thermodynamics but all of them are equivalent, it is possible that most of the entropic methods applied to the nervous system and brain in particular share a similar outcome, or essence, that helps understand some aspects of the fundamentals of basic neurodynamics. In this short review focused on certain aspects of the entropic metrics some results are examined that indicate the fundamental importance of the natural tendency towards a maximal energy distribution for healthy brain activity and thus cognition to emerge.
\end{abstract}

Keywords - Entropy Information Nervous system Energy Cognition

\section{Introduction}

Entropic measures have been applied to the nervous system in many shapes and forms. Considering the almost ethereal nature of entropy (didn't von Neumann advise Shannon to call his measure entropy because "nobody knows what entropy really is?") these various applications to a number of measured variables (ions, neuronal connections, neurophysiological signals, etc.) pose the risk of confusion, of misleading the interpretations and thus the research to understand nervous system function.

Caution should be taken when comparing results from diverse entropy applications. Even when it is a similar type of entropy (say, Shannon entropy) that is applied to different variables -ions diffusing through membranes, network connectivity etc.- the results may not be comparable at all, each one will stand on its own and will have a specific interpretation. The ideal situation would be if a common essence could be extracted, distilled, from the very different applications to a variety of observables. In the same manner we cannot use a man-made metric like weight measured by a balance to directly infer the volume occupied by that weight (ten kilograms of tomatoes occupy more volume than the same weight of iron), care should exercised when directly inferring and comparing interpretations from entropy applied to a wide variety of observables.

The purpose of this text is an attempt at clarification of what some entropic measures applied to experimentally obtained variables mean and at the same time to try to distill the essence of these applications, to find a common theme that improves the understanding of the nervous systems and perhaps glimpse at the emergence of cognition. Hence, we will not focus on very specific technical applications of entropic metrics to particular nervous system variables but rather the emphasis will be on the neurophysiological meaning of some studies that may reveal fundamentals of basic neurodynamics.

Finally, these results here surveyed suggest that the common theme underlying many entropic measures revolves around the well-known tendency of natural phenomena towards a maximal energy distribution -nothing more than the second principle of thermodynamics- which in terms of the nervous system is manifested by maximisation of the number of configurations of cellular contacts or connections.

\section{The many faces of entropy}

As aforementioned, entropy has been applied to a variety of variables recorded, or obtained, from the nervous systems. If Thurner et al. described the three faces of entropy in the analysis of complex systems [1], here we present a concise survey of some aspects of nervous system activity that have been used for diverse entropy methods, the main purpose being to exhibit the many applications and some results but without going deep into any of these (there are so many that an entire encyclopedia would be needed!). 
However, and along the lines of Thurner etal, let us briefly review the three main different manners to define entropy. The first one is the notion of entropy developed in Physics in the mid-19th century. Clausius introduced the notion of thermodynamic entropy $S$ as an extensive quantity that links temperature with heat [2, 3. Later, Boltzmann was able to relate this thermodynamic entropy to the number of microstates $\Omega$ in a system

$$
S_{B}=k \ln \Omega
$$

where $S$ is the entropy, $k$ is the Boltzmann's constant and $\Omega$ the number of possible microstates of the system. The equation assumes that all microstates have the same probability of occurrence. A microstate is a particular configuration of the components of a system that is consistent with the macroscopic quantities that characterize the system (such as volume, pressure or temperature). Independently from physics, in the context of information theory (IT), a functionally identical notion of entropy appears in the work of Claude Shannon 4]. In IT, $H$ of a random variable is the average level of "information", "surprise", or "uncertainty" inherent in the variable's possible outcomes. The information production rate $S_{I T}=H$ determines if information can be coded, transmitted through a noisy channel and decoded in an error-free way. Given a discrete random variable $X$, with possible outcomes $x_{1}, \ldots, x_{n}$, which occur with probability $P\left(x_{1}\right), \ldots, P\left(x_{n}\right)$, the entropy of $X$ is formally defined as:

$$
S_{I T}(X)=-\sum_{i=1}^{n} P\left(x_{i}\right) \log P\left(x_{i}\right)
$$

where $\Sigma$ denotes the sum over the variable's possible values, the choice of base varying between different applications. Finally, Jaynes developed the maximum entropy principle (MEP) [5] as a method to address statistical inference problems that are not confined to physics. The principle of maximum entropy $\left(S_{M E P}=H\right)$ states that the probability distribution which best represents the current state of knowledge is the one with largest entropy, in the context of precisely stated prior data. Since the distribution with the maximum entropy is the one that makes the fewest assumptions about the true distribution of data, the MEP can be seen as an application of Occam's razor. While these are the three main notions of entropy, one has to always keep in mind that in the final analysis entropy represents energy distribution, it is an index of energy dispersal within a system and between the system and its surroundings 6 .

Having briefly reviewed the main notions of entropy, let us describe some applications in neuroscience. In the neurological context, at the microscopic level, entropy has been applied to characterise time dependent signals in sequences of discrete, identical action potentials or spikes, the information being carried in the spike arrival time and entropy is used in this context to quantify this information without any assumption about which feature of the spike train is more important 7]. Following this line of thought, other authors have studied the upper limit of the information content of a single spike using Landauer's principle, the results indicating that an action potential can process more information than a single bit of the Shannon entropy [8]. Entropy has been applied as well to the molecular world, e.g., a mathematical model for ion diffusion in ion channels studied aspects of ion diffusion contributing to entropy production 9]. Synapses have not escaped either to the application of entropic metrics, for instance addressing the balance between excitatory and inhibitory synapses needed to ensure appropriate function of neural networks, study that demonstrated highest entropy at the boundary between excitation-dominant and inhibition-dominant regimes [10]; as well, correlation entropy has been applied to synaptic input-output dynamics showing that cortical synapses exhibit low-dimensional chaos driven by natural input patterns [11].

At the macroscopic scale, entropy calculations have been very widely used to analyze electroencephalographic (EEG) signals, be it scalp EEG or intracranial/intracerebral EEG, and of course magnetoencephalographic (MEG) recordings and functional neuroimaging (fMRI) signals. The vast majority of computations are based on Shannon entropy and differ about which method is used to discretize the signal, e.g. binarization, histograms or wavelets. A widely used measure is the so-called permutation entropy (PE), which is a robust time series tool that provides a quantification of the complexity of a dynamic system capturing the order relations between values in the time series and then extracting a probability distribution of the ordinal patterns [12. The PE is based on Shannon entropy but others entropies have been used, such as Renyí or Tsalli's entropy [13, 14. It has had a myriad applications in the analysis of signals ranging from epilepsy and anaesthesia to Alzheimer's disease [15 17]. There are modifications of these methods that take into account the length of the signal [18] or that give weights to the amplitude of the signal [19].

Other entropy measures such as Spectral entropy (SE) have been popular too in the analysis of neural signals at the collective level (those abovementioned EEG, MEG and similar recordings that record the activity of large cell ensembles). $\mathrm{SE}$ is a Shannon entropy measure over the spectral power distribution of the signal. It treats the signal's normalized power distribution in the frequency domain as a probability distribution, and calculates the Shannon entropy of it; the power frequency domain can be obtained using the Fourier [20], Hilbert 21] or wavelet transform (called Wavelet entropy) 22]. A major, practical application is in the analysis of EEG signals under anaesthesia to assess the level of patient sedation; these studies have shown that as sedation increases the entropy values associated with the EEG signals decrease [20]. As well, SE has been used in the study of sleep stages 23] and in the detection of epileptiform activity [24]. The wavelet entropy, which has been proposed as a metric to evaluate brain rhythms using event related potentials [25], has been applied to assess recovery after brain injury [26] and in Alzheimer's disease [27.

Functional neuroimaging data, such as functional magnetic resonance, have been subject to entropy measurements in many studies. As illustrations, it has been used to characterize ageing properties of the human brain through the analysis of resting state blood oxygen level dependent (BOLD) signals, indicating that the functional entropy associated with brain activity increases with age 28; as well, a link between human intelligence and brain entropy has been sought in a study 
that found that brain entropy was positively associated with intelligence [29], and in the abstract of the paper the authors mention that "This relationship between high brain entropy and high intelligence indicates an essential role for entropy in brain functioning". It may be worth pointing out now one aspect, perhaps a warning, about all these entropy estimations that will be addressed in following sections, namely, that in reality rather than entropy having a "role in" some event, it is the physical meaning of the measure -energy distribution, see section 44, its underlying physical substrate that has the role, because one should not forget that entropy is a measure we have devised to characterise natural phenomena, but whether it can be considered a cause of processes driving living systems is a matter of debate [30. It will be emphasised in sections below that entropy is an index of energy dispersal [6] and thus energy gradients are the fundamental essence directing the evolution of natural phenomena. We close this brief overview of entropy applications to nervous system observable with a mention of studies using sample entropy applied to fMRI in subjects with attention deficit hyperactivity disorder, revealing abnormalities in various brain regions [31, and in patients with schizophrenia where more complex signal patterns were found in the patients as compared to healthy individuals [31]. And finally, an interesting study used the Shannon entropy estimated from BOLD fMRI data obtained in subjects using psychedelic compounds, and found that this entropy increases in the psychedelic state, thereby resulting in a greater repertoire of connectivity motifs among brain networks in the psychedelic state than during normal waking consciousness 32 . This result is incorporated into the Entropic Brain hypothesis which proposes that, within certain bounds, the entropy of spontaneous brain activity indexes the richness of conscious states 33 .

Overall, the results in many of these studies suggest that, in general, higher entropy is related to healthier brain function although care has to be taken with the physiological interpretation because, as mentioned below, entropy can be applied to a variety of observable and its interpretation may vary. Considering entropy as an index of energy dispersal, or dissipation, it is therefore plausible that energy is better distributed in healthy conditions and during conscious awareness. We will examine this proposal in the next sections.

\section{Entropy as an information metric: energetic considerations}

Entropy has been very widely employed as a metric of information. The matter of information to characterise nervous system function has been taken with great enthusiasm since times past, which is reasonable because, as W. Bialek mentioned: "The generation of physicists who turned to biological phenomena in the wake of quantum mechanics noted that, to understand life, one has to understand not just the flow of energy but also the flow of information. There is, of course, some difficulty in translating the colloquial notion of "information" into something mathematically precise." 34. Indeed, brain information processing has been used many times in the literature in its widest, intuitive sense (for a general account of what is conceived by brain computation and information processing see [35) and while it is true that the concept of information has several connotations and is somewhat relative, the notion of information derived from entropy is more or less well characterised and understood, thanks to the works of C. Shannon and others. It has been the Shannon framework that has been taken by several investigators to address questions about neural coding. Let us recall that in the Shannon formalism entropy should be conceived as a measure on a probability distribution characterizing, so to speak, the amount of missing information that distribution represents.

Therefore, at a microscopic scale, the neuronal action potentials (spikes) have been treated in some works as binary electrochemical switches -1 when the neuron fires the spike and 0 when it is at rest 36 38. Since the probability that a neuron will fire an action potential is influenced by many factors such as firing threshold, temperature, presynaptic inputs, degree of connectivity and so forth, these two states can be treated as a random variable with two possible states $X_{f}$ (firing) and $X_{r}$ (resting). The probability of occurrence for each one is represented as $P_{f}=P\left(X_{f}\right)$ and $P_{r}=P\left(X_{r}\right)$ respectively, satisfying $P_{f}+P_{r}=1$; we can then write $P_{r}=1-P_{f}$ and replacing in Shannon's formula 2 we obtain

$$
S=-P_{f} \cdot \log \left(P_{f}\right)-\left(1-P_{f}\right) \cdot \log \left(1-P_{f}\right)
$$

That is, the entropy of this system depends on the probability of the neuron firing. This framework has been widely applied to address the question of neural coding. For instance, to compare the information transferred in particular neurocomputational models of the stimulus-response function, showing that dynamic stimuli can be encoded efficiently by single neurons and that each spike contributes to information transmission [39].

Similar analysis could be made at a macroscale level defining the states of the system as the pairwise connections between brain areas (or, to be more precise, between brain signals recorded from the tissue) 40. In this case, the random variable becomes the connectivity between two networks: "connected", $X_{c}$, or "disconnected", $X_{d c}$, with the associated probabilities $P_{c}$ and $P_{d c}$ respectively. Connections in these cases meaning functional connections, that is, any metric like a synchrony index that is high enough, above a certain threshold, to consider the two networks (signals) as "connected". As before, we can write the entropy in function of probabilities of connections

$$
S=-P_{c} \cdot \log \left(P_{c}\right)-\left(1-P_{c}\right) \cdot \log \left(1-P_{c}\right)
$$

In both cases the graphic of the entropy $S$ is an inverted $\mathrm{U}$, reaching the peak at $P_{f}=P_{r}=1 / 2$, or at the macroscale case at $P_{c}=P_{d c}=1 / 2$; This maximum in the curve represents the maximum uncertainty of the system, or, in the statistical mechanics framework, the maximization of the possible (micro)states of the system (for methodological aspect see A.

But it is the relation of the Shannon formalism with the energy distribution or dispersal that interests us here, because entropy is not really a measure of disorder or chaos as commonly taught [6] -even though in some specific contexts entropy 
is reflecting disorder, but not always- entropy should not be used to estimate the degree of disorder in self-organising systems [41]. Entropy represents energy distribution. Therefore rather than asserting that entropy is a driving force, it could be more reasonable to assert that energy diffusion, the energy dissipation or dispersion in a final state compared to an initial state is the driving force, entropy just being the index of that dispersal within a system and between the system and its surroundings [6]. Let us emphasise that we are not declaring that entropy plays no role on driving natural phenomena, rather what is meant is that entropy is a sort of arbitrary measurement that can be applied to many observables and hence the interpretation may vary; on the other hand, considering entropy as the dispersion or distribution of energy, then it is that dissipation of energy what can be thought of as the driving force of events. Hence, in reality one concept (more distribution of energy) is equivalent to the other (higher entropy), the main advantage using the energy perspective is that it is less ambiguous than the possible arbitrariness of the entropic viewpoint.

The relation between energy and entropy or information has been the subject of study for many decades [42]; indeed it was Clausius' original intention to relate energy and entropy -thermal entropy being part of the total energy content measuring how much of that energy becomes useless. Many scholars have further contributed fundamental notions on the topic, e.g., the Landauer's principle connecting the information theoretic notion of entropy to statistical mechanics. Let us note in our context that in the same manner as the equiprobability of states maximise the Shannon entropy (equation 2 ), the equal distribution of energy among the possible states maximise too that entropy. To see this is trivial: maximal entropy occurs for equiprobable events $P_{i}=1 / N$ (say, of a total $N$ states), then using Boltzmann's approach (we have omitted the partition function and will assume that $\left.k_{B} T=1\right): P_{i}=e^{-E_{i}}$ and $P_{i}=1 / N$ then $1 / N=e^{-E_{i}}$ hence $E_{i}=\log (N)$ all states have same energy. Therefore increasing entropy entails increasing energy distribution among the microstates. One can as well substitute energy for probability in Shannon's formula yielding $S \sim \sum_{i}=e^{-E_{i}} \cdot E_{i}$ which serves to perform analyses relating directly the energy of the microstates to the entropy $S$, but this is of course beyond the scope of our review article. The important matter to discuss here is what this equal energy dispersal among the microstates may reveal or say about brain dynamics. The next section expounds the possible neurophysiological interpretation of this result in light of past experiments and analyses done on brain recordings.

\section{Entropic measures applied to the brain networks' collective ac- tivity: maximization of configurations of network connections for proper 1 brain function.}

Let us consider now what entropy applied to the study of the brain coordination dynamics -the cellular collective activity at the meso or macroscale level- may reveal in terms of possible fundamental principles of brain organization from where cognition and conscious awareness emerge. The reason to emphasise the cellular collective dynamics to understand the integrative functions of nervous systems is exemplified in the words of the neurologist and polymath Oliver Sacks: "That the brain is minutely differentiated is clear: there are hundreds of tiny areas crucial for every aspect of perception and behavior... The miracle is how they all cooperate, are integrated together, in the creation of the world. This, indeed, is the problem, the ultimate question, in neuroscience..." [43. It is therefore important to explore at a relatively high level of description that cooperation among brain cellular ensembles, for it is known that each neuron's activity is meaningful only with respect to other cells' actions; the foundation of neural information processing lies in the interaction between cells, and this is the reason why so many studies are devoted to the assessment of the patterns of neuronal organized activity using metrics like synchrony, coherence, correlations and similar methods. Cognition is after all a pattern of organised activity in the (embodied) nervous system, paraphrasing E.J. Ambrose's words "Life is basically a pattern of organized activity" 44].

Results derived from the estimation of the entropy associated with the number of possible configurations of pairwise brain network connectivity indicate that brain macrostates associated with conscious awareness possess more microstates (in terms of configurations of network connections) and thus higher entropy than unconscious states [40, 45]. These studies used invasive and non-invasive electrophysiological brain recordings in different states of consciousness (sleep, wakefulness, seizures and coma) and the phase synchrony between pairs of signals was evaluated, then a threshold value of the synchronization index was used to determine when two signals were "connected" (see section A for notes on the putative "connection" between brain networks/signals). Each state of consciousness studied was considered a macrostate of brain synchronization, composed of a number of microstates which are the several possible configurations of the connected signals.

We clarify that the states talked about here are the microstates of synchronised (or connected) brain regions that all together form the whole state, the macrostate, and that there is no assumption that a macrostate is associated with a particular mental state unless in simple cases like all-to-all connectivity where there is only one microstate forming the macrostate -which could represent an epileptic seizure where all nets are synchronized. While no connection is made with the psychological level of mental states, it is conceivable that the global mental macrostate during normal wakefulness corresponds to the combination of all that sensorimotor processing carried out by the different microstates. These studies used pair-wise interactions of signals, but the relevance of other high-order interactions has been demonstrated in theoretical neuroscience as paired-based analysis sometimes does not provide enough information to study a system.

\footnotetext{
${ }^{1}$ By proper, or optimal brain function we refer to that working of neural network which is adaptive to the organism, an appropriate processing of sensorimotor transformations.
} 
The entropy applied to the distribution of cellular connectivity, neural synchronization and similar aspects are therefore related to the characterization of cellular interactions, how the activity of cell networks are organised, but activity is after all energy. Hence, the essence of the entropy applied to most of these variables detecting cellular activity is about energy distribution. The finding that entropy associated with the number of the configurations of brain networks is largest during states of conscious awareness $[40,45]$ is an indication that energy is maximally distributed among those networks in these behavioural states. Following thermodynamic considerations about the time evolution of the entropy, energy dissipation was found larger during conscious awareness as opposed to unconscious pathological states -seizures and coma [30].

From the perspective of what entropy really signifies, an index not of disorder but of energy distribution, the observation of larger entropy associated with the configurations of neuronal network connections during conscious awareness as opposed to unconscious states has a clear neurophysiological interpretation. It is not that brain activity is more disorganised or random during wakefulness, which is counterintuitive, rather it means that energy (that is, cellular activity) is distributed in more ways. Voluntary motor actions or any mental event for that matter involve the coordinated activity of many neurons in many areas - organised activity after all- so for the sake of simplicity let us imagine there are 100 neuronal chains each able to process one input of the many that an individual receives during the waking state. Each of those chains can be envisaged as an energy gradient, from the first to the last neuron in the chain. Thus, establishing many energy gradients is an optimal brain organization for the adaptability of the organism to a changing environment: in our simple example, it can process 100 inputs simultaneously. Now let us take one clear example of lack of responsiveness: absence seizures; in these cases the thalamus and the cortex are mutually entrained due to their recurrent anatomical connectivity, so it can be pictured as just one energy gradient, from the thalamus to the cortex. One neuronal chain, one energy gradient, is not optimal to process a myriad of inputs, hence the unresponsive state ensues. Therefore, it is all about the number of ways in which energy can be dispersed. Depicted in Figure 1 is the main concept summarising the experimental results aforementioned where conscious awareness was associated with the largest number of configurations of connections among cell networks or brain areas; that is, there are more possible ways energy can be dispersed as the number of connectivity configurations increases, and the maximum of ways occurs at the top of the inverted $U$ (in the original publications that curve was the entropy associated with the number of configurations). In pathological cases like coma, energy is in fact dispersed as well, but as there is almost no variability in neural connectivity in coma (same would apply to epileptic seizures), then the ways to distribute that energy become much reduced. It is therefore in this sense we talk about "energy dispersal", basically a shorthand for "greater variability in connectivity that is helpful for brains to operate".

Therefore the tendency to maximise energy distribution/dissipation emerges from those entropic measures. This maximisation is naturally subjected to constraints, including the neuroanatomical connectivity, the total energy available and the inputs the brain receives in diverse behavioural states; basically, it depends on the context, the situation where the brain rests. Taking the aforesaid perspective that the brain microstates represent configurations of "connected" brain cell ensembles -it has been proposed that the nature of brain states are patterns of coordinated activity that can be observed as correlations/synchronization of activity in cell ensembles [30]- then the comment in the previous section on the equal distribution of energy among microstates maximising entropy finds here its neurophysiological correlate: energy, that is the neural activity, tends to be distributed as widely as possible in normal waking consciousness and the result of that energy distribution is the maximisation of brain microstates, the configurations of connectivity patterns among brain networks.

But these are not novel concepts. Scholars since times past have voiced the notion that living processes make the energy flux through the system a maximum, of course so far as compatible with constraints [46, and that the flow of energy through a system acts to organise that system [47. While these early thoughts were mostly concerned with metabolic, living systems, the idea can be adapted to the nervous system because just like metabolic pathways offer channels for energy to flow, neural communication provides further conduits for that flow. It has been proposed that the expression of this feature of maximising the energy flow/distribution in the function of the brain could be the main factor accountable for the emergence of conscious awareness. But one should always be careful about using extremal principles such as maximisation or minimisation of entropy for nonequilibrium processes [41]. As well, consideration has to be given to the fact that processes like energy distribution/dispersal/dissipation are bounded by environmental constraints.

Nevertheless, considering the possibility of the tendency towards maximization of energy spread, this perspective could be more effective than talking about maximization of man-made metrics like entropy or information. In this regard, some scholars are reluctant to consider maximization principles as cause of processes driving living systems [48, view which may be correct because concepts like information or entropy are, in the final analysis, man-made creations developed to characterize certain phenomena; energy, on the other hand, seems to be a more fundamental aspect of natural phenomena, although here too efforts at obtaining precise and strict definitions of energy have not yielded too much success, the definitions proposed being inherently circular (see [4, chap 12]). In the final analysis, it is the gradients of energy, the energy dissipation in a final state compared to an initial state, the driving force, and the entropy is the index of that dispersal [6].

Other entropic measures used in various studies are related as well to the number of configurations of brain network connections. For example, permutation entropy applied to electroencephalographic recordings differentiates periods of epileptic seizures versus interictal activity -activity without seizures [13]-, or sample entropy applied to resting state functional neuroimaging reveals a positive correlation between high entropy and intelligence in healthy individuals [29]. Both sample entropy and permutation entropy measure the predictability of time series, the latter more concerned with the temporal ordering structure of the series, which in the end all reflect the variability in neural network connections from where the brain signals are recorded; in other words, the essence is again the neural collective activity patterns, or the energy distribution among brain cell ensembles. Equivalently, Shannon entropy applied to probability distributions of 


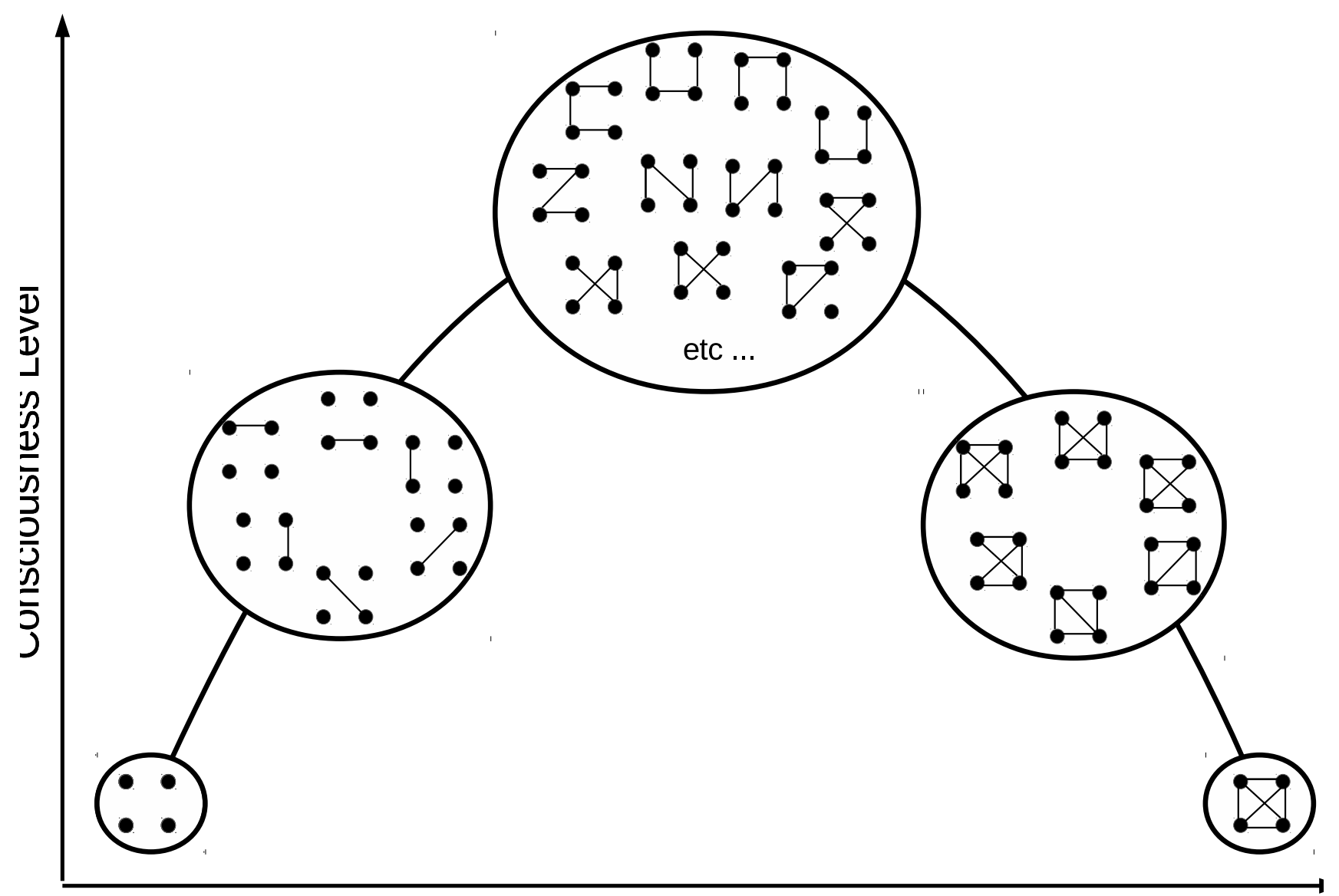

Nlimbor of nonnontod hroin arnon

Figure 1: Proposed general scheme of the relation between global brain connectivity and consciousness level. Shown are 4 neuronal networks (dots) and the microstates (connectivity patterns) forming the macrostate (circles) due to some possible connection patterns. For no connections (lower left) and all-to-all connection (lower right), there is only one configuration possible, one microstate. As connections are allowed, more configurations are possible (that is, starting from the lower left). The top of the curve represents the higher number of possible configurations that the system can adopt, in this case of 4 networks occurring when there are 3 connections. The maximization of the configurations (microstates) provides the variability in brain activity and a diversity of paths for energy transfer among networks needed for normal sensorimotor action. On the other hand, large or small number of connected networks exhibiting lower number of microstates (hence lower entropy) are not optimal for sensorimotor processing, giving abnormal or unconscious states. 
brain network synchrony over time -again reflecting configurations of activity or energy distribution- revealed a greater collection of functional brain connectivity motifs in the psychedelic state as compared to waking consciousness as was mention in section 2 [32].

For the sake of completion, it should be noted that the ideas behind how the information flow is related to energy flows have been treated in some studies [50] and have been the subject of special issues, e.g. "Information and Energy/Matter" [51. As well, a recent interest in considerations from the energy viewpoint has emerged, for instance in the free energy principle for biological systems [52].

\section{$5 \quad$ On nervous system criticality}

Before concluding this brief overview on entropy applied to neural systems, a few words on the concept of critical dynamics and how this relates to the results reviewed in this text. Basically, criticality stems from the competition between the ordering tendency of the interactions among the system constituents and the disordering tendency of (stochastic) fluctuations; or, from another perspective, the redistribution of energy among the system constituents results in critical phenomena. It has been proposed that the collective organization of cellular activity in brains possess features of critical dynamics in order to mimic what is found out there in nature, which is normally described by critical dynamics 53 . To be fair, let us note that there still remains debate as to whether nervous systems actually operate close to criticality -commonly assessed using power law distributions- or in another state such as oscillatory or stochastic dynamics; some studies have indicated that critical and stochastic dynamics occur at the same time in the brain 54. It has to be considered as well that features associated with criticality emerge in situations even when the dynamics are not critical, e.g. in networks with a hierarchical modular structure [55].

In any event, this criticality entails a large number of metastable states -microstates that appear and disappearthus flexible patterns of communication are possible among brain cell ensembles that allow the nervous system to process information in an adaptive fashion: the more patterns the better to process myriads of incoming stimuli, although of course the patterns have to be organised, coordinated somehow, hence this field of study is normally referred to as brain coordination dynamics [56. Incidentally, while the notion of instability and metastability of brain dynamics is relatively recent, one of the very early proposals of the brain as an "organ whose natural state is one of unstable equilibrium" is due to William James in 1879 [57.

A good deal of work has been done investigating transitions between the brain metastable states. A dynamic complex system which has multiple coexisting stable states and can move from one to another in response to either noise or intrinsic perturbations to the system is generally called a metastable system [58, 59. Particularly, the brain "resting state" is not a homogeneous state but a period of switching among multiple micro-states, and thus it could be considered as an example of metastability. 60, 61. To address the question of the state transitions between these metastable microstates in brain dynamics, the concepts of energy landscape (EL) analysis have recently been applied to fluctuations of blood oxygenation level-dependent BOLD fMRI 62 66. The main idea behind EL analysis in the study of brain dynamics is the description of brain states as distributed activity patterns across brain regions [66]. The EL of the system is formed by several valleys with local minima called "stable states" that have energies lower than their neighbours. Results using this EL framework indicated that the cerebral cortex at rest is a more complex and organised state transition network than "altered" states such as sleep [67. As can be seen, then, the energy perspective has been useful to determine state transition in brain activity, and underlying the EL framework is the concept of energy redistribution among these states.

It is thus adaptability the key feature that the maximisation of connectivity configurations confers the organism. Illustrations of this fact are the reports that lower entropy associated with the number of configurations of connections was found when visual input was diminished -subjects with eyes closed [40]- and a less complex network structure was manifest as compared with eyes open condition [68. Hence the results on the maximisation of entropy mentioned in section 4 apply in reality more to an appropriate brain information processing than to consciousness, for one is very conscious when his/her eyes are closed and yet the entropy associated with network connections was lower. Can this variability of interactions among neural nets, these fluctuations in connectivity patterns, be a most fundamental organising principle of nervous system function? A direct test for this proposal requires the quantification of those fluctuations in, say, neuronal synchronization or similar observables characterising functional connections. Several results, too many to be covered here, suggest this may be the case, which led to the proposal of a possible scenario for the transition from healthy brain activity towards pathological activity [69.

\section{Concluding remarks}

Systems tend to attain the most probable state -equilibrium, which, incidentally is a concept closely connected with that of observation time and thus somewhat relative [70]- which normally consists of a macrostate represented by the largest number of microstates. In the case of brains supporting cognition, proper brain function requires an efficient web of neural connections, which, interpreted from the perspective expounded in this text, is equivalent to saying that nervous systems tend to a widespread energy distribution among all those microstates via maximization of the number of configurations. In chemistry, for instance, one talks about the number of molecular configurations in chemical reactions; in the case of brains the macrostates consist of the number of configurations of connections among neural networks; brain macrostates associated with conscious awareness possess more microstates, or configurations of connections [45], whose emergence and 
dissolution determine cognitive states. In other words, the embodied brains that are immersed in an environment seem to attempt to reach equilibrium, which is not possible due to the exchange of energy (information) with the environs.

Since we have now considered the concept of equilibrium, it is important to clarify that, as stated above, equilibrium is a relatively arbitrary notion that depends on context and the level of description. The essence of the notions of the approach to equilibrium - and the easiest manner to comprehend what this means- is to think about it as the approach to the most probable state. This is too what the famous increase in entropy observed in natural phenomena represents: the tendency of natural phenomena to approach the most probable state. In our studies, as mentioned above, we found that the macrostate made up of the largest number of microstates -the most probable macrostate- occurs during conscious awareness and we refer to this as equilibrium. But it depends on context, on the constraints imposed by the environment, because when the context determines a diminution of sensory inputs, such as when falling asleep, then there appears another most probable state that can be thought of as another equilibrium, this one determined by the specific features of thalamic and cortical neurons and their connections that cause the typical slow wave rhythms during deep sleep; this equilibrium lasts about 4 to 8 hours (depending on how much one likes to sleep). Hence, depending on the context, the brain will try to approach its most likely state. The equilibrium notion depends on the level of description too: at the microscopic, molecular level, we normally find in the body non-equilibrium situations (ion gradients, nonlinear action potentials etc.), but at the macro level we find the body at near equilibrium, it is called homeostasis: constant temperature, blood pressure etc. Hopefully now it has become clearer what equilibrium we are discussing in this text and the relevance of realising how arbitrary this concept is.

It is fair to mention that the theme expounded in this text about brain macrostates associated with conscious awareness possessing more microstates (in terms of configurations of network connections) and thus higher entropy than unconscious states, is related to other proposals for frameworks that characterise brain function; specifically, the information integrated theory (IIT) 71] the global workspace theory (GWT) 72], and the metastability of brain states framework [7] all have as an underlying notion the need for a substantial number of microstates -configurations of network connections in our textin order to integrate information (as postulated by the IIT), to broadly broadcast activity to various brain regions (as proposed by the GWT) and to avoid becoming trapped in one stable activity pattern (as the metastability notion states).

The emergence of cognition due to nervous systems maximising energy distribution through connectivity patterns is probably a feature not only of humans but also of most animals. Although there have not been observations directly related to this proposal in experiments in other animals as those reported above in the case of human recordings during various conscious states, but considering the continuum in the development of cognition, we propose that this key feature is present in most living organisms [74, 75,

The intricate web of neuronal assemblies giving rise to a myriad of energy gradients may result from the recently proposed "neuroglial closure" in brain cell circuits [76]; when this closure, this wide interconnectedness, disappears transiently, for instance during epileptic seizures, the consequence is loss of conscious awareness. And generally speaking this is maximisation of energy distribution is a natural tendency, just like gas molecules tend to occupy the maximal volume available, energy will redistribute in the widest manner for no particular reason but because that is the most probable outcome. The diverse natural phenomena and their specific mechanisms are details as to how the optimal energy distribution is achieved, and from the variety of these mechanisms and phenomena our concepts of order/disorder and stochasticity/determinism, emerge in attempts to describe them.

\section{A A note on some methodological aspects and what these may reveal about brains.}

It is worth mentioning that in the process of the analysis done in the aforesaid estimation of entropy associated with the number of configurations of brain network connectivity a threshold value of the phase synchronization index (derived from the analytic signal concept, see [77]) was used to determine when two signals were "connected" [40, 45]. We will not delve into the issue of whether measuring correlations between signals (phase synchrony, coherence, cross-correlations etc.) reflect "real" connectivity, as this matter has been treated in numerous texts; suffice to say here that phase synchrony was taken as an indication of connections because when two brain networks have synchronous activity this indicates that they are probably involved in processing the same input or performing same sensorimotor action (there is a very extensive literature on anatomical and functional connectivity concepts and methods of evaluation). The synchrony threshold was taking from surrogates - that is, from random time series, or, to be more precise, the phase of the signals was randomisedderived from the original recordings. Interestingly, the average value of the phase synchrony index from the surrogate population was nearly identical to the value of the index obtained from signals in the waking state. This means, basically, that brain activity during normal wakefulness, at least in terms of synchronization, is equivalent to that of random activity (for those not conversant with what surrogates are in this type of research, let us just say in simple words that they represent random, or pseudo-random - see comments below- signals obtained from the originals and are used to assess when synchrony is "real" or just accidental). The observation that the synchrony among normal brain signals do not differ that much from that of random signals has been reported in various studies [78; here we show a quantitative illustration: the average of the phase synchrony index in a montage of intracerebral EEG (iEEG) taken in a subject during normal waking consciousness was $0.399 \pm 0.03$, and that of the surrogate population was $0.394 \pm 0.01$, and in a monkey (a close relative) intracranial recordings (ECoG) had a synchrony index average of $0.393 \pm 0.02$, and $0.391 \pm 0.08$ for the surrogates 
But what does this mean, for brain function and cognition in general?

First let us note that the notion of randomness is a very relative concept. To wit, number $e$ (or $\pi$ ) may be random or deterministic depending on how it is "measured". Statistical tests applied to the sequence of digits will indicate randomness. But we know how to generate those digits using a formula, in case of $e$ one formula is $e=\lim [1+1 / n]^{n}$ hence it is not random but totally determined by the mathematical expression. Hence, is $e$ random or deterministic? It depends on the viewpoint. Having said this, the fact that surrogate signals provide similar synchrony as those during waking consciousness is a consequence of the signals measured, which at the mesoscale level reflect the activity of large cellular populations, and considering that the brain has to integrate and segregate a myriad of sensorimotor transformations simultaneously in order to adequately perceive and respond to incoming stimuli $[30,76]$, then this neuronal activity at the microscale level is observed at higher levels as stochastic. It does not mean a healthy brain displays random activity, rather it needs to support so many different configurations of micro-level connections (the aforesaid large entropy during conscious states) that depending on the measurement it will appear as organised or as purely stochastic; if we were to follow the activity in specific microcircuits we would see organised activity, but when the recordings sample thousands of these organised microcircuits the result is a noisy, random-like time series. Therefore, a noisy brain is a healthy brain [79].

\section{Conflict of Interest Statement}

The authors declare that they have no conflict of interest.

\section{References}

[1] Stefan Thurner, Bernat Corominas-Murtra, and Rudolf Hanel. Three faces of entropy for complex systems: Information, thermodynamics, and the maximum entropy principle. Physical Review E, 96(3):32124, 2017.

[2] S N Carnot. Reflexions sur la puissance motrice du feu et sur les machines propres a developper cette puissance(Reflections on the Motive Power of Fire), Paris, 1824. In Annales scientifiques de l'École Normale Supérieure, pages $393-457$.

[3] Rudolf Clausius. Über eine veränderte Form des zweiten Hauptsatzes der mechanischen Wärmetheorie. Annalen der Physik, 169(12):481-506, 1854.

[4] C Shannon. A mathematical theory of communication. Bell System Technical Journasth, 27(3):379-423, 1948.

[5] E T Jaynes. Information theory and statistical mechanics. Physical review, 106(4):620, 1957.

[6] Frank L Lambert. Disorder-A cracked crutch for supporting entropy discussions. Journal of Chemical Education, $79(2): 187,2002$.

[7] S P Strong, Roland Koberle, Rob R de Ruyter van Steveninck, and William Bialek. Entropy and Information in Neural Spike Trains. Physical Review Letters, 80(1):197-200, 1998.

[8] S. Street. Upper Limit on the Thermodynamic Information Content of an Action Potential. Preprints, 2020020035, 2020.

[9] A Gomez-Mellado, T Munoz, C Paiva, Karina Vilches, and I Carvacho. Entropy production of ion thermo-diffusion in cell membranes. In Journal of Physics: Conference Series, volume 1160, page 12013. IOP Publishing, 2019.

[10] Vidit Agrawal, Andrew B Cowley, Qusay Alfaori, Daniel B Larremore, Juan G Restrepo, and Woodrow L Shew. Robust entropy requires strong and balanced excitatory and inhibitory synapses. Chaos: An Interdisciplinary Journal of Nonlinear Science, 28(10):103115, 2018.

[11] Ingo C Kleppe and Hugh P C Robinson. Correlation entropy of synaptic input-output dynamics. Physical Review E, 74(4):41909, 2006.

[12] Christoph Bandt and Bernd Pompe. Permutation entropy: a natural complexity measure for time series. Physical review letters, 88(17):174102, 2002.

[13] Nadia Mammone, Jonas Duun-Henriksen, Troels Kjaer, and Francesco Morabito. Differentiating interictal and ictal states in childhood absence epilepsy through permutation Rényi entropy. Entropy, 17(7):4627-4643, 2015.

[14] Zhenhu Liang, Yinghua Wang, Xue Sun, Duan Li, Logan J Voss, Jamie W Sleigh, Satoshi Hagihira, and Xiaoli Li. EEG entropy measures in anesthesia. Frontiers in Computational Neuroscience, 9, feb 2015.

${ }^{2} \mathrm{~A}$ quick note on the phase synchrony index computation. Pairwise phase synchrony (calculated as in [77, 78] was evaluated for 46 iEEG channels and for 128 ECoG channels. Signals were pre-processed in the standard fashion and filtered using a frequency band of $28 \pm 2 \mathrm{~Hz}$. To compute the synchrony index for the surrogate population, 10 surrogates for each original signal were created and same procedure for the index computation was applied, using a 1 second running window and obtaining the average index from the total number of pairwise combinations. 
[15] Xiaoli Li, Gaoxian Ouyang, and Douglas A Richards. Predictability analysis of absence seizures with permutation entropy. Epilepsy research, 77(1):70-74, 2007.

[16] N Nicolaou and J Georgiou. The Use of Permutation Entropy to Characterize Sleep Electroencephalograms. Clinical EEG and Neuroscience, 42(1):24-28, 2011.

[17] F C Morabito, D Labate, F La Foresta, A Bramanti, G Morabito, and I Palamara. Multivariate multi-scale permutation entropy for complexity analysis of Alzheimer's disease EEG. Entropy, 14(7):1186-1202, 2012.

[18] Wajid Aziz and Muhammad Arif. Multiscale Permutation Entropy of Physiological Time Series. In 2005 Pakistan Section Multitopic Conference. IEEE, 2005.

[19] B Fadlallah, B Chen, A Keil, and J Príncipe. Weighted-permutation entropy: A complexity measure for time series incorporating amplitude information. Physical Review E, 87(2):22911, feb 2013.

[20] A Vakkuri, A Yli-Hankala, P Talja, S Mustola, H Tolvanen-Laakso, T Sampson, and H Viertio-Oja. Time-frequency balanced spectral entropy as a measure of anesthetic drug effect in central nervous system during sevoflurane, propofol, and thiopental anesthesia. Acta Anaesthesiologica Scandinavica, 48(2):145-153, feb 2004.

[21] Xiaoli Li, Duan Li, Zhenhu Liang, Logan J Voss, and Jamie W Sleigh. Analysis of depth of anesthesia with HilbertHuang spectral entropy. Clinical Neurophysiology, 119(11):2465-2475, nov 2008.

[22] O A Rosso, S Blanco, J Yordanova, V Kolev, A Figliola, M Schürmann, and E Bacsar. Wavelet entropy: a new tool for analysis of short duration brain electrical signals. Journal of neuroscience methods, 105(1):65-75, 2001.

[23] Jürgen Fell, Joachim Röschke, Klaus Mann, and Cornelius Schäffner. Discrimination of sleep stages: a comparison between spectral and nonlinear EEG measures. Electroencephalography and Clinical Neurophysiology, 98(5):401-410, may 1996.

[24] N Kannathal, Min Lim Choo, U Rajendra Acharya, and P K Sadasivan. Entropies for detection of epilepsy in EEG. Computer methods and programs in biomedicine, 80(3):187-194, 2005.

[25] R Quian Quiroga, O A Rosso, E Baar, and M Schrmann. Wavelet entropy in event-related potentials: a new method shows ordering of EEG oscillations. Biological Cybernetics, 84(4):291-299, mar 2001.

[26] Hasan A Al-Nashash, Joseph S Paul, Wendy C Ziai, Daniel F Hanley, and Nitish V Thakor. Wavelet Entropy for Subband Segmentation of EEG During Injury and Recovery. Annals of Biomedical Engineering, 31(6):653-658, jun 2003.

[27] Functional disorganization of small-world brain networks in mild alzheimer's disease and amnestic mild cognitive impairment: an eeg study using relat@articlefrantzidis2014functional, title=Functional disorganization of small-world brain networks in mild Alzheimer's Disease and amnestic Mild Cognitive Impairment: an EEG study using Relative Wavelet Entropy (RWE),ive Wavelet Entropy (RWE), author=Frantzidis, Christos A and Vivas, Ana B and Tsolaki, Anthoula and Klados, Manousos A and Tsolaki, Magda and Bamidis, Panagiotis D, journal=Frontiers in aging neuroscience, volume $=6$, pages $=224$, year $=2014$, publisher $=$ Frontiers.

[28] Y Yao, W L Lu, B Xu, C B Li, C P Lin, D Waxman, and J F Feng. The Increase of the Functional Entropy of the Human Brain with Age. Scientific Reports, 3(1), oct 2013.

[29] Glenn N Saxe, Daniel Calderone, and Leah J Morales. Brain entropy and human intelligence: A resting-state fMRI study. PloS one, 13(2), 2018.

[30] Jose Luis Perez Velazquez, Diego Martin Mateos, and Ramon Guevara Erra. On a simple general principle of brain organization. Frontiers in Neuroscience, 13:1106, 2019.

[31] Moses O Sokunbi, Wilson Fung, Vijay Sawlani, Sabine Choppin, David E J Linden, and Johannes Thome. Resting state fMRI entropy probes complexity of brain activity in adults with ADHD. Psychiatry Research: Neuroimaging, 214(3):341-348, 2013.

[32] Robin Lester Carhart-Harris, Robert Leech, Peter John Hellyer, Murray Shanahan, Amanda Feilding, Enzo Tagliazucchi, Dante R Chialvo, and David Nutt. The entropic brain: a theory of conscious states informed by neuroimaging research with psychedelic drugs. Frontiers in human neuroscience, 8:20, 2014.

[33] Robin L Carhart-Harris. The entropic brain-revisited. Neuropharmacology, 142:167-178, 2018.

[34] William Bialek. Biophysics: searching for principles. Princeton University Press, 2012.

[35] Gualtiero Piccinini and Andrea Scarantino. Information processing, computation, and cognition. Journal of biological physics, 37(1):1-38, 2011. 
[36] Fred Rieke, David Warland, Rob R de Ruyter van Steveninck, and William Bialek. Spikes: Exploring the Neural Code. Press, MIT, 1999.

[37] Peter Sterling and Simon Laughlin. Principles of Neural Design. The MIT Press, jun 2015.

[38] Jonathan D Victor. Approaches to Information-Theoretic Analysis of Neural Activity. Biological Theory, 1(3):302316 , sep 2006.

[39] Alexander Borst and Frédéric E Theunissen. Information theory and neural coding. Nature neuroscience, 2(11):947957, 1999.

[40] R Guevara Erra, D M Mateos, R Wennberg, and J L Perez Velazquez. Statistical mechanics of consciousness: Maximization of information content of network is associated with conscious awareness. Physical Review E, 94(5):52402, 2016.

[41] Leonid M Martyushev and Vladimir D Seleznev. Maximum entropy production principle in physics, chemistry and biology. Physics reports, 426(1):1-45, 2006.

[42] Myron Tribus and Edward C McIrvine. Energy and information. Scientific American, 225(3):179-190, 1971.

[43] Oliver Sacks. An anthropologist on Mars: Seven paradoxical tales. Vintage, 2012.

[44] Edmund Jack Ambrose. The nature and origin of the biological world. Technical report, 1982.

[45] D M Mateos, R Wennberg, R Guevara, and J L Perez Velazquez. Consciousness as a global property of brain dynamic activity. Physical Review E, 96(6):62410, 2017.

[46] Alfred J Lotka. Contribution to the energetics of evolution. Proceedings of the National academy of Sciences of the United States of America, 8(6):147, 1922.

[47] Harold Morowitz and Eric Smith. Energy flow and the organization of life. Complexity, 13(1):51-59, 2007.

[48] Hermann Haken. Information and self-organization: A macroscopic approach to complex systems. Springer Science and Business Media, 2006.

[49] Paul L Nunez. Brain, mind, and the structure of reality. Oxford University Press, 2012.

[50] Pedro C Marijuán, Jorge Navarro, and Raquel del Moral. How prokaryotes 'encode'their environment: Systemic tools for organizing the information flow. Biosystems, 164:26-38, 2018.

[51] Gordana Dodig Crnkovic. Information and energy/matter, 2012.

[52] Friston Karl. A free energy principle for biological systems. Entropy, 14(11):2100-2121, 2012.

[53] D R Chialvo. Emergent complex neural dynamics. Nature physics, 6(10):744-750, 2010.

[54] Jesse Tinker and Jose Luis Perez Velazquez. Power law scaling in synchronization of brain signals depends on cognitive load. Frontiers in Systems Neuroscience, 8, may 2014.

[55] Eric J Friedman and Adam S Landsberg. Hierarchical networks, power laws, and neuronal avalanches. Chaos: An Interdisciplinary Journal of Nonlinear Science, 23(1):13135, 2013.

[56] J A Scott Kelso. Dynamic patterns: The self-organization of brain and behavior. MIT press, 1995.

[57] W M JAMES. I. - ARE WE AUTOMATA? Mind, os-4(13):1-22, 1879.

[58] J A Scott Kelso. Multistability and metastability: understanding dynamic coordination in the brain. Philosophical Transactions of the Royal Society B: Biological Sciences, 367(1591):906-918, 2012.

[59] Jean-Luc Schwartz, Nicolas Grimault, Jean-Michel Hupé, Brian C J Moore, and Daniel Pressnitzer. Multistability in perception: binding sensory modalities, an overview, 2012.

[60] Michael Breakspear. Dynamic models of large-scale brain activity. Nature neuroscience, 20(3):340-352, 2017.

[61] Mikhail I Rabinovich and Pablo Varona. Robust transient dynamics and brain functions. Frontiers in computational neuroscience, 5:24, 2011.

[62] Jiyoung Kang, Chongwon Pae, and Hae-Jeong Park. Energy landscape analysis of the subcortical brain network unravels system properties beneath resting state dynamics. NeuroImage, 149:153-164, 2017.

[63] Takamitsu Watanabe, Satoshi Hirose, Hiroyuki Wada, Yoshio Imai, Toru Machida, Ichiro Shirouzu, Seiki Konishi, Yasushi Miyashita, and Naoki Masuda. Energy landscapes of resting-state brain networks. Frontiers in neuroinformatics, $8: 12,2014$. 
[64] Takamitsu Watanabe, Shigeyuki Kan, Takahiko Koike, Masaya Misaki, Seiki Konishi, Satoru Miyauchi, Yasushi Miyahsita, and Naoki Masuda. Network-dependent modulation of brain activity during sleep. NeuroImage, 98:1-10, 2014 .

[65] Takahiro Ezaki, Michiko Sakaki, Takamitsu Watanabe, and Naoki Masuda. A ge-related changes in the ease of dynamical transitions in human brain activity. Human brain mapping, 39(6):2673-2688, 2018.

[66] Shi Gu, Matthew Cieslak, Benjamin Baird, Sarah F Muldoon, Scott T Grafton, Fabio Pasqualetti, and Danielle S Bassett. The energy landscape of neurophysiological activity implicit in brain network structure. Scientific reports, 8(1):1-15, 2018.

[67] Jiyoung Kang, Chongwon Pae, and Hae-Jeong Park. Graph-theoretical analysis for energy landscape reveals the organization of state transitions in the resting-state human cerebral cortex. PloS one, 14(9):e0222161, 2019.

[68] Marie-Therese Horstmann, Stephan Bialonski, Nina Noennig, Heinke Mai, Jens Prusseit, Jörg Wellmer, Hermann Hinrichs, and Klaus Lehnertz. State dependent properties of epileptic brain networks: Comparative graph-theoretical analyses of simultaneously recorded EEG and MEG. Clinical Neurophysiology, 121(2):172-185, 2010.

[69] Jose Luis Perez Velazquez and Marina Frantseva. The Brain-Behavior Continuum: The Subtle Transition Between Sanity and Insanity. World Scientific, 2011.

[70] Craig Callender. Taking thermodynamics too seriously. Studies in History and Philosophy of Science Part B: Studies in History and Philosophy of Modern Physics, 32(4):539-553, 2001.

[71] G Tononi. An information integration theory of consciousness. BMC neuroscience, 5(1):42, 2004.

[72] B J Baars. A cognitive theory of consciousness. Cambridge University Press, 1993.

[73] K J Friston. Book Review: Brain Function, Nonlinear Coupling, and Neuronal Transients. The Neuroscientist, 7(5):406-418, 2001.

[74] Robert E Ulanowicz. Ecological Clues to the Nature of Consciousness. Entropy, 22(6):611, 2020.

[75] John Stewart. Cognition= life: Implications for higher-level cognition. Behavioural processes, 35(1-3):311-326, 1995.

[76] Jose Luis Perez Velazquez. On the emergence of cognition: from catalytic closure to neuroglial closure. Journal of Biological Physics, pages 1-25, 2020.

[77] F Mormann, K Lehnertz, P David, and C E Elger. Mean phase coherence as a measure for phase synchronization and its application to the EEG of epilepsy patients. Physica D: Nonlinear Phenomena, 144(3):358-369, 2000.

[78] Luis Garcia Dominguez, Richard A Wennberg, William Gaetz, Douglas Cheyne, O Carter Snead, and Jose Luis Perez Velazquez. Enhanced synchrony in epileptiform activity? Local versus distant phase synchronization in generalized seizures. Journal of neuroscience, 25(35):8077-8084, 2005.

[79] Andrea B Protzner, Taufik Valiante, Natasa Kovacevic, Cornelia McCormick, and Mary Pat McAndrews. Hippocampal signal complexity in mesial temporal lobe epilepsy: a noisy brain is a healthy brain. Archives italiennes de biologie, 148(3):289-297, 2010. 